\title{
A Special Issue on Geothermal Energy
}

\author{
Roland Horne • Kewen Li
}

Published online: 12 December 2014

(C) International Association for Mathematical Geosciences 2014

It is a pleasure for us to introduce this Special Issue of Mathematical Geosciences on "Geothermal Energy", dedicated to all members of the worldwide geothermal community.

Geothermal energy is clean, indigenous, and base-load renewable energy, not subject to intermittencies of weather and time-of-day. In recent years, great advances have been achieved in reservoir modeling, geology, geophysics, geochemistry, and other areas of geosciences. These achievements have contributed to steady growth of the geothermal industry. Facing the rapid evolution of the field, the goal of this special issue is to gather some of the recent contributions about methods and theories relevant to the geosciences of finding and developing geothermal energy.

The first of the six papers on this Special Issue is by Jonny Rutqvist, Patrick F. Dobson, Julio Garcia, Craig Hartline, Pierre Jeanne, Curtis M. Oldenburg, Donald W. Vasco, and Mark Walters who deal with the prestimulation modeling and interpretation of the stimulation in the northwest Geysers EGS (Enhanced Geothermal System) demonstration project, California. EGS is one of the hot spots in geothermal research in recent years. EGS has a huge potential. It is clear that EGS is presently still at the "proof of concept" stage. Without question, EGS is currently a great challenge for geothermal industry. However, these authors were able predict the extent of the stimulation zone reasonably well. Their modeling data indicate that the microearthquake events are related to shear reactivation of preexisting fractures that are triggered by the combined effects of injection-induced cooling around the injection well and small changes in steam pressure.

Many geothermal reservoirs, including EGS, are fractured systems. It is important to characterize the heat transfer in such fractured reservoirs. Suzuki et al. derived a

R. Horne $(\varangle) \cdot$ K. Li

Stanford Geothermal Program, Stanford University, Stanford, CA, USA

e-mail: horne@stanford.edu 
new heat transfer model combined with the fractional advection dispersion equation that describes non-Fickian dispersion in the fractured reservoir. They then analyzed the mechanism of water injection in fractured reservoirs using a fractional-derivativebased mass and heat transfer model.

Ames et al. developed a new method for characterizing temperature distributions in geothermal reservoirs using the threshold reactive tracers (nanoreactors). They demonstrated through modeling that the location of temperature measurements could be determined to map the interwell temperature profile at a given time.

Characterization of fracture systems is a great challenge, so many researchers have been searching for solutions. In this regard, Alghalandis et al. proposed the connectivity field as a useful measure to evaluate the spatial connectivity characteristics of fractures in a fracture network. The connectivity field can be used to determine the optimal location of injection or production wells so as to maximize reservoir performance.

Along a similar line, Magnusdottir and Horne's paper proposes a new approach to estimating fracture connectivity in geothermal reservoirs using time-lapse electric potential data. They verified the advantages of using electric potential measurements instead of only the tracer return curves.

The Special Issue concludes with the topic of geothermal resources coproduced from oil and gas reservoirs, in a paper by Li and Sun. Coproduction of geothermal energy from petroleum formations may be a type of resource that we could turn into real power capacity in the near term, if EGS is a hope in the longer term. In this paper, $\mathrm{Li}$ and Sun modified the existing volumetric approach to evaluate the geothermal resource in oil and gas reservoirs by considering the hydrocarbon phase saturations and the change with time. The effect of oil and/or gas saturations on the geothermal resource may be neglected when the porosity is less than a specific value (about $5 \%$ ).

We hope that you will enjoy the papers of this Special Issue! 